\title{
SELF-HEALING THERMOPLASTIC-TOUGHENED EPOXY
}

\author{
A. Jones ${ }^{1}, \mathrm{~S}$. White ${ }^{2,3}$ and N. Sottos ${ }^{2,4}$ \\ 1 Department of Mechanical Science and Engineering, University of Illinois at Urbana- \\ Champaign, 1206 W. Green St, Urbana, Illinois 61801, USA - e-mail: emnett1@illinois.edu \\ ${ }^{2}$ Beckman Institute for Advanced Science and Technology, 405 N. Mathews, Urbana, Illinois \\ 61801, USA - e-mail: swhite@illinois.edu; n-sottos@illinois.edu \\ ${ }^{3}$ Department of Aerospace Engineering, University of Illinois at Urbana-Champaign, $104 \mathrm{~S}$. \\ Wright St, Urbana, Illinois 61801, USA - e-mail: swhite@illinois.edu \\ 4 Department of Material Science and Engineering, University of Illinois at Urbana- \\ Champaign, 1304 W. Green St, Urbana, Illinois 61801, USA-e-mail: n-sottos@illinois.edu
}

Keywords: self-healing, thermoplastic-toughening, polymer-matrix composites, solvent

\begin{abstract}
Microcapsule based self-healing strategies enable repair of matrix cracking and delamination in fiber reinforced composite materials. Most of the encapsulated healing systems reported to date lack stability at high temperatures and limit composite processing to relatively low cure temperatures. In prior literature, successful crack healing has been achieved in high temperature cured composites by incorporation of a thermoplastic phase in the matrix. These systems are not autonomous and require the application of heat to soften the thermoplastic phase and initiate healing. In this work, a thermoplastic resin is blended with a high performance epoxy matrix to simultaneously toughen and act as a healing agent in combination with encapsulated solvents.

Self-healing is achieved for a commercial Epon 828 epoxy resin cured with diamino diphenyl sulfone above $150^{\circ} \mathrm{C}$. Based on early screening studies, two thermoplastic toughening agents, poly bisphenol A-co-epichlorohydrin and polysulfone, are identified as model thermoplastic/ epoxy systems to study. Each system is evaluated using a tapered double cantilever beam (TDCB) fracture specimen geometry. The effect of cure cycle, thermoplastic loading, thermoplastic type, and capsule loading on both phase separation and healing response is investigated. To achieve in situ healing, microcapsules containing a polymer co-dissolved in a solvent are added to the thermoplastic-epoxy blend. Key parameters for capsule stability, such as capsule core content, shell wall robustness, and maximum cure temperature, are identified.

Additionally, the optimized systems from the TDCB study are extended to repair of interfacial damage between the fiber and matrix. The effect on both healing performance and interfacial bond strength due to different fiber sizings and interfacial phase separation is investigated. Since interfacial debond of reinforcing fibers is one of the key failure mechanisms in composite materials, prevention of growth of these flaws could greatly extend the lifetime of the composite.
\end{abstract}

